\title{
Résolution sur le renforcement de la coopération entre les États pour lutter contre les faux médicaments et les produits médicaux falsifiés
}

\author{
$13^{\mathrm{e}}$ Conférence des chefs d'État et de gouvernement des pays ayant \\ le français en partage. Montreux (Suisse), 23-24 octobre 2010
}

\section{Resolution on the reinforcement of inter-state cooperation against counterfeit drugs and adulterated medical products}

13th Conference of the Heads of State and Government of Francophone countries.

Montreux (Switzerland), 23-24 October 2010

Nous, chefs d'État et de gouvernement des pays ayant le français en partage, réunis les 23 et 24 octobre 2010 à Montreux, en Suisse, à l'occasion du $13^{\mathrm{e}}$ Sommet de la francophonie :

- rappelant que le droit de jouir d'un meilleur état de santé physique et mentale est l'un des droits fondamentaux de tout être humain ;

- rappelant également les Objectifs du millénaire pour le développement et plus particulièrement ceux liés à la santé ;

- constatant que la production, la mise en circulation et la vente de faux médicaments et de produits médicaux falsifiés ainsi que l'accroissement de ce trafic constituent une grave menace pour la santé publique, en particulier dans les pays en développement ;

- réaffirmant le rôle fondamental que joue l'OMS pour veiller à ce que l'innocuité, la qualité et l'efficacité des produits médicaux soient assurées ;

- considérant la nécessité de mettre un terme à cette situation inacceptable ;

- reconnaissant la mobilisation internationale qui découle de l'Appel de Cotonou, qui a fait suite à la Conférence internationale sur les faux médicaments tenue à Cotonou le 12 octobre 2009 ;

- convaincus qu'une action efficace contre les faux médicaments et les produits médicaux falsifiés exige le renforcement de la coopération entre les États et la coordination entre toutes les autorités concernées, médicales, policières, douanières et judiciaires.
Nous engageons à :

- nous mobiliser de façon urgente pour faire de la lutte contre les faux médicaments et les produits médicaux falsifiés une priorité et développer des politiques visant à assurer la disponibilité de médicaments et de produits médicaux de qualité, sûrs, efficaces et d'un prix abordable pour les populations les plus démunies, en particulier dans les pays en développement ;

- demander aux instances internationales de continuer à soutenir la mise en oeuvre d'actions concertées plurisectorielles, tant au niveau national, régional qu'international ;

- renforcer les capacités humaines et techniques dans le domaine de la lutte contre les faux médicaments et les produits médicaux falsifiés, optimiser les moyens d'intervention et sécuriser le circuit de distribution des médicaments et des produits médicaux, notamment en renforçant les capacités des laboratoires de contrôle de qualité ;

- participer pleinement et activement aux travaux menés au sein de l'OMS, dans le cadre de la lutte contre les produits médicaux falsifiés, en vue d'un renforcement de la coordination et de la coopération internationales en la matière;

- soutenir, dans le cadre des initiatives internationales existantes, la tenue de conférences nationales et régionales visant à intensifier la coopération en la matière et à aboutir à terme à l'élaboration d'instruments juridiques sur la lutte contre les faux médicaments et les produits médicaux falsifiés. 